ARTICLE

https://doi.org/10.1038/s41467-019-10838-1

\title{
Atomic-scale perturbation of oxygen octahedra via surface ion exchange in perovskite nickelates boosts water oxidation
}

\author{
Jumi Bak ${ }^{1}$ Hyung Bin Bae ${ }^{2}$ \& Sung-Yoon Chung (iD ${ }^{1}$
}

A substantial amount of interest has been focused on $\mathrm{ABO}_{3}$-type perovskite oxides over the past decade as oxygen electrocatalysts. Despite many studies on various compositions, the correlation between the structure of the oxygen octahedra and electrocatalytic property has been overlooked, and there accordingly have been a very limited number of attempts regarding control of atomistic structure. Utilizing epitaxial $\mathrm{LnNiO}_{3}(\mathrm{Ln}=\mathrm{La}, \mathrm{Pr}, \mathrm{Nd}$ ) thin films, here we demonstrate that simple electrochemical exchange of $\mathrm{Fe}$ in the surface region with several-unit-cell thickness is notably effective to boost the catalytic activity for the oxygen evolution reaction by different orders of magnitude. Furthermore, we directly establish that strong distortion of oxygen octahedra at the angstrom scale is readily induced during the Fe exchange, and that this structural perturbation permits easier charge transfer. The findings suggest that structural alteration can be an efficient approach to achieve exceptional electrocatalysis in crystalline oxides.

\footnotetext{
${ }^{1}$ Department of Materials Science and Engineering, Korea Advanced Institute of Science and Technology (KAIST), 291 Daehak-ro, Yuseong-gu, Daejeon 34141, Korea. ${ }^{2}$ KAIST Analysis Center, Korea Advanced Institute of Science and Technology (KAIST), 291 Daehak-ro, Yuseong-gu, Daejeon 34141, Korea. Correspondence and requests for materials should be addressed to S.-Y.C. (email: sychung@kaist.ac.kr)
} 
O xygen evolution and reduction are the major electrochemical reactions in electrolysers, metal-air batteries, fuel cells, and water-splitting devices, and facilitating these reactions has been a central issue for effective fuel generation and higher energy conversion/storage efficiency ${ }^{1-5}$. It is generally accepted that the activation barrier of the oxygen evolution and reduction reactions is fundamentally large and that multiple intermediate steps of electron transfer are necessarily involved during the reactions. Adequate electrocatalysts are thus essential for promoting the reactions at lower overpotentials. In particular, since both the oxygen evolution reaction (OER) and the oxygen reduction reaction (ORR) in metal-air batteries and water-splitting devices take place at room temperature ${ }^{1,3}$, enhancing the oxygen electrocatalysis has a crucial impact not only on the efficiency of such devices, but on their longevity and ultimate performance.

Since the milestone works by Bockris and Otagawa ${ }^{6,7}$ in the early 1980s, substantial attention has been paid to employing perovskite oxides as OER electrocatalysts with the aim of replacing expensive precious-metal-based materials such as $\mathrm{IrO}_{2}$ and $\mathrm{RuO}_{2}{ }^{3,8,9}$. Furthermore, sparked by recent findings on the notably high OER catalytic activity of a multiple-cation perovskite ${ }^{10}$, there has been strong interest in $\mathrm{ABO}_{3}$-type perovskite oxides as alternative OER catalysts ${ }^{11-20}$. Plausible mechanisms have been reasonably suggested, based on the $B-\mathrm{OH}$ bond strength (where $B$ is a transition metal) ${ }^{21-23}$, the $3 d$-shell electronic configuration $^{10}$, the position of the $\mathrm{O} 2 p$ band center in the electronic band structure $^{11}$, the degree of hybridization between $B$-site metal and oxygen orbitals ${ }^{14,24}$, and the charge-transfer energy between $B$ and $\mathrm{O}^{18,25}$. Although there is no single universal descriptor for OER electrocatalysis using perovskite oxides ${ }^{9}$, it is recognized in general that the electronic structure of the $\mathrm{O} 2 p$ and metal $3 d$ bands strongly correlates with the OER catalytic activity.

A significant change can be induced in the electronic structure of both the metals and the oxygen in many oxides by varying their physical structure, including by lattice strain ${ }^{26}$. Accordingly, one of the crucial initial steps toward establishing the link between the crystal structure, the electronic configurations, and the overall OER catalytic performance is the precise identification of changes in the surface structure by direct observation at the atomic scale $27-29$. From a structural viewpoint, the $A B \mathrm{O}_{3}$ perovskite framework consists of corner-shared $\left[\mathrm{BO}_{6}\right]$ octahedral units, which are catalytically active sites ${ }^{7}$, together with $A$-centered dodecahedra. Consequently, identifying variations in the shape of the $\left[\mathrm{BO}_{6}\right]$ octahedra and understanding under what circumstances structural change takes place remain key issues for the accurate prediction of electronic structure and subsequent OER activity. In particular, since the shape of the $\left[\mathrm{BO}_{6}\right]$ octahedra is determined largely by the location of oxygen anions in the lattice, visualization of the oxygen positions is an essential aspect of the direct observation of structure, despite being very challenging.

By utilizing (001) epitaxial thin films of lanthanide nickelate perovskites, $L n \mathrm{NiO}_{3}$ (where $\mathrm{Ln}$ is $\mathrm{La}, \mathrm{Pr}$, and $\mathrm{Nd}$ ), in this work we demonstrate that the structural perturbation of metal-oxygen octahedra via simple electrochemical Fe exchange at the surface is particularly efficient at enhancing OER activity, by an order of magnitude. To conduct atomic-column resolved observation of oxygen displacement, we used integrated differential phasecontrast (iDPC) scanning transmission electron microscopy $(\mathrm{STEM})^{30,31}$, a recently developed phase-contrast imaging technique, in addition to well-known annular bright-field (ABF) STEM. Electron energy-loss spectroscopy (EELS) and energydispersive X-ray spectroscopy (EDS) were also carried out to verify the chemical exchange of the Fe for the Ni site. Combined with ab initio density functional theory (DFT) calculations, the experimental findings in the present study provide significant evidence showing that a high density of states of $\mathrm{O} 2 p$ and transition-metal $3 d$ orbitals near the Fermi level can be achieved by the strong distortion of the oxygen octahedra, boosting the OER activity in the perovskite nickelates.

\section{Results}

Fe incorporations in $\mathrm{LaNiO}_{3}$ thin films. As demonstrated in $\mathrm{Ni}$ based oxides and (oxy)hydroxides, Fe is known to be an effective solute cation that can significantly enhance OER activity ${ }^{32-41}$. We accordingly prepared two different types of (001) thin-film $\mathrm{LaNiO}_{3}$ samples for comparison. One was a simple solid-solution homogeneously containing $\mathrm{Fe}$ in the lattice by high-temperature annealing during film fabrication. Figure la shows a $10 \%-\mathrm{Fe}-$ doped $\mathrm{LaNiO}_{3}$ solid-solution film deposited on a (001) $\mathrm{SrTiO}_{3}$ single-crystal substrate. Both the high-angle annular dark-field (HAADF) STEM image and a set of EDS chemical maps in Fig. 1a clearly verify the homogeneous distribution of Fe over the entire film, in addition to epitaxial growth with high crystallinity (see Supplementary Fig. 1 for a series of X-ray diffraction patterns and atomic EDS maps showing the solid-solution of Fe and the epitaxial growth). The other type are $\mathrm{LaNiO}_{3}$ thin films that contain Fe exclusively in the surface region, induced via electrochemical ion exchange, which will be described in more detail below. Both sets of EDS maps in the middle column of Fig. 1b, c show the presence of $\mathrm{Fe}$ confined to the surface region. Atomic-scale HAADF images also reveal that there is no structural decomposition at the film surface, indicating that the $\mathrm{Fe}$ has been substituted for $\mathrm{Ni}$ as solute atoms. To more precisely determine the depth of $\mathrm{Fe}$ exchange, we obtained electron-energy loss spectrum images using the $\mathrm{Fe}-L_{3}$ peak during the EELS analysis. Based on the Fe maps shown in the right-hand column of Fig. 1b, c, a distinct amount of $\mathrm{Fe}$ is detectable above the background noise from several unit cells below the surface, not merely from the topmost surface (see Supplementary Fig. 2 for two series of $\mathrm{Fe}-L_{3}$ peaks).

Surface Fe exchange. The Fe exchanges demonstrated in Fig. 1b, c were achieved by electrochemical methods at room temperature. One approach adopted for the sample shown in Fig. 1b is schematically depicted in Fig. $2 \mathrm{a} . \mathrm{Ni}^{3+}$ is strongly driven to become $\mathrm{Ni}^{2+}$ under reducing conditions in a $\mathrm{KOH}$ aqueous solution far below $1.23 \mathrm{~V}$ vs. reversible hydrogen electrode (RHE), thereby resulting in the exsolution of $\mathrm{Ni}$ from the perovskite lattice. Indeed, when the reduction reaction was repeated many times, we could identify the formation of a seriously Ni-deficient amorphous surface layer, directly demonstrating the Ni extraction (see Supplementary Fig. 3a). At the same time, nanoscale Nibased oxide (or hydroxide) precipitates, possibly in the form of $\mathrm{Ni}$ $(\mathrm{OH})_{2}$ (or NiO) (see Supplementary Fig. 3b) were also found on the surface after 20 -cycle reduction reactions down to $0.62 \mathrm{~V}$ vs. RHE. As a result, if the number of reactions was adequately controlled at five cycles, $\mathrm{Ni}$ vacancies could be created in the surface region without collapsing the crystalline perovskite framework in the surface region. As $\mathrm{Ni}$ vacancies are effectively negatively charged defects $\left(V_{\mathrm{Ni}}{ }^{\prime \prime \prime}\right)$, electron holes $\left(h^{\bullet}\right)$ confined in the $\mathrm{O} 2 p$ orbitals hybridized with the $\mathrm{Ni} 3 d$ orbitals are anticipated to form in order to satisfy the overall charge neutrality during the pre-reduction reaction (see Supplementary Note 1 for more details, including the Mulliken electronic population analysis). After this pre-reduction, a cyclic potential between 1.27 and $1.75 \mathrm{~V}$ vs. RHE was applied to the sample in a Fe-containing $\mathrm{KOH}$ solution so that $\mathrm{Fe}^{3+}$ would fill the empty $\mathrm{Ni}$ sites, as illustrated in Fig. 2a. The high-magnification HAADF image and 

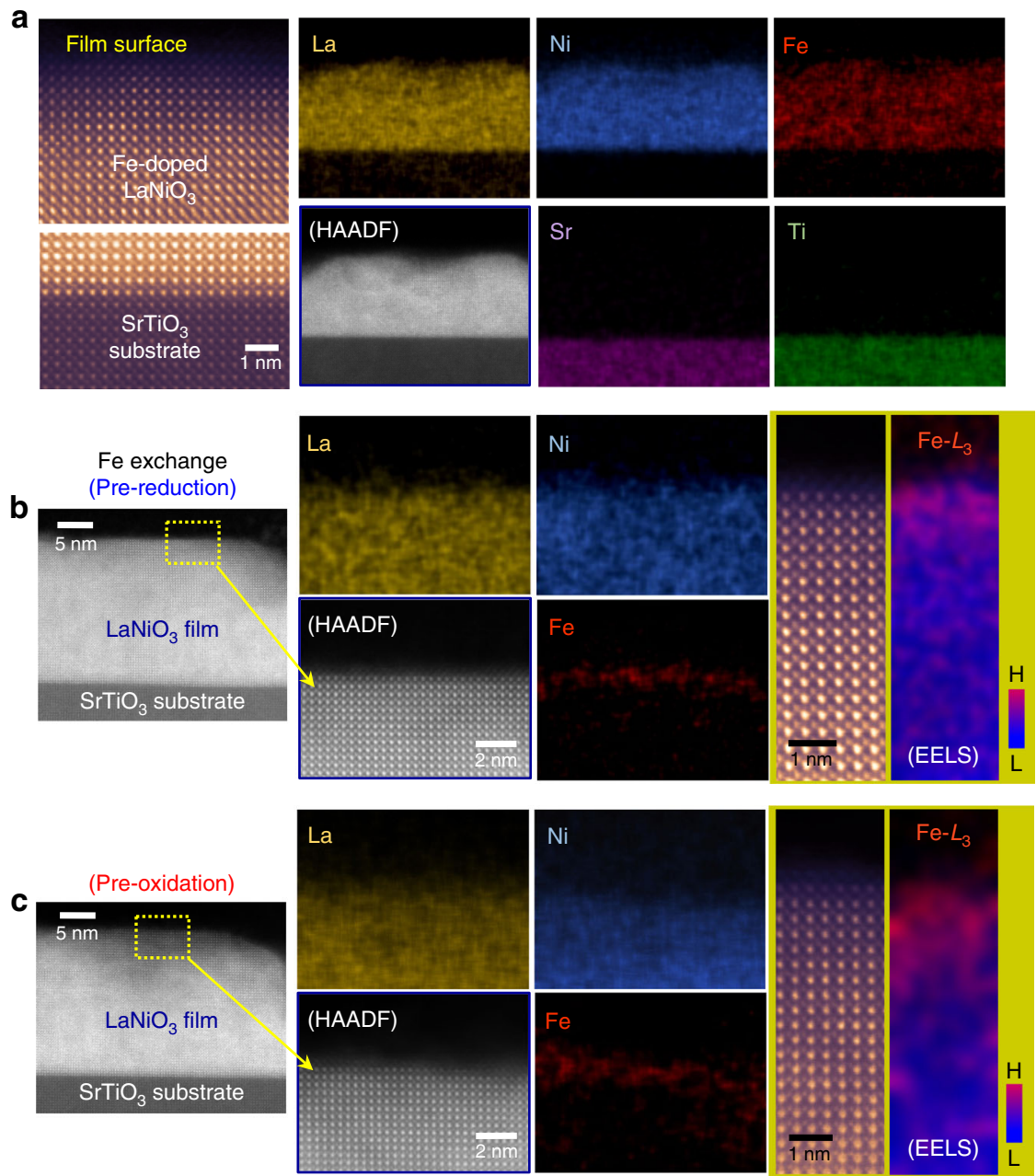

Fig. $1 \mathrm{Fe}$ incorporations in $\mathrm{LaNiO}_{3}$ epitaxial thin films. All the thin films were fabricated on (001) $\mathrm{SrTiO}_{3}$ substrates. a The composition of the film is La $\left(\mathrm{Ni}_{0.9} \mathrm{Fe}_{0.1}\right)_{3}$. This set of energy-dispersive $\mathrm{X}$-ray spectroscopy (EDS) maps clearly shows the homogenous distribution of Fe over the entire film, verifying a solid-solution. The high-angle annular dark-field (HAADF) image in the left-hand column proves the high crystallinity and epitaxial growth of the film. b, c These $\mathrm{LaNiO}_{3}$ films were subjected to surface Fe exchange by pre-reduction (b) and pre-oxidation (c) reactions. The presence of Fe in each surface region is confirmed by both EDS and electron energy-loss spectroscopy (EELS) mapping. In particular, the EELS maps and corresponding atomic-column images in the right-hand column show that a detectable amount of Fe is observed down to 3-5 unit cells below the surface

the Fe EELS map in Fig. 1b demonstrate that this pre-reduction step is very efficient at promoting ion exchange with Fe.

The other approach to achieve $\mathrm{Ni}$ extraction is based on oxidation reactions. We experimentally found that $\mathrm{Ni}$ cations from $\mathrm{LaNiO}_{3}$ thin films could dissolve into a $\mathrm{KOH}$ electrolyte solution at high oxidation potentials. Like the previous prereduction, a remarkably $\mathrm{Ni}$-deficient amorphous layer could be identified at the film surface after 20-cycle oxidation reactions up to $1.85 \mathrm{~V}$ vs. RHE (see Supplementary Fig. 4). As before, when this pre-oxidation step was limited to several cycles to preserve the crystalline framework of the films, Fe exchange could be effectively achieved by filling empty $\mathrm{Ni}$ sites with $\mathrm{Fe}^{3+}$ from the electrolyte solution. This process is schematically described in Fig. $2 \mathrm{~b}$ and was experimentally verified by STEM, as shown in Fig. 1c. Oxygen anions in the lattice can evolve at a high overpotential under the oxidizing condition, resulting in the formation of oxygen vacancies. It is thus reasonably anticipated that the negatively charged $\mathrm{Ni}$ vacancies $\left(V_{\mathrm{Ni}^{\prime \prime}}{ }^{\prime \prime}\right)$ originating from the $\mathrm{Ni}$ dissolution are compensated by the positively charged oxygen vacancies $\left(V_{O} \cdot{ }^{*}\right)$ as Schottky-type defects during the preoxidation reaction (see Supplementary Note 2 for more details). Additional evidence obtained by EDS showing the surface Fe exchange through the pre-reduction and the pre-oxidation steps is provided in Supplementary Fig. 5.

As the Ni- $L_{3}$ peak in EELS considerably overlaps with the La- $M_{4}$ peak, it is fairly difficult to track variations in the $\mathrm{Ni}-L_{3}$ peak intensity in $\mathrm{LaNiO}_{3}$ without rigorous deconvolution of the two peaks ${ }^{42}$. Although a detectable amount of $\mathrm{Fe}$ was clearly probed $\sim 3$ unit cells beneath the surface in Fig. $1 \mathrm{~b}$ and $\sim 5$ unit cells in Fig. 1c using EELS, it was not possible to verify the change in $\mathrm{Ni}$ content in the $\mathrm{LaNiO}_{3}$ thin films. We therefore fabricated epitaxial thin films of other nickelates, $\mathrm{PrNiO}_{3}$ and $\mathrm{NdNiO}_{3}$, where the Ni- $L$ peaks do not overlap with $\mathrm{Pr}-$ and $\mathrm{Nd}-M$ peaks, on (001) $\mathrm{SrTiO}_{3}$ substrates (see Supplementary Fig. 6 for details on the films). Figure $2 \mathrm{c}$ shows two series of $\mathrm{Fe}-L$ and Ni- $L$ peaks acquired in the epitaxial $\mathrm{PrNiO}_{3}$ film following $\mathrm{Fe}$ exchange via the pre-oxidation method. As denoted by numbers in the atomicscale HAADF image in the right-hand column, each peak was collected approximately at a unit-cell interval. The two sets of EELS peaks demonstrate the increase in $\mathrm{Ni}$ intensity and a simultaneous decrease in $\mathrm{Fe}$ intensity with the peak collection below the surface. This directly proves the replacement of $\mathrm{Ni}$ with Fe. Consistent sets of $\mathrm{Fe}-L$ and $\mathrm{Ni}-L$ peaks obtained in the epitaxial $\mathrm{NdNiO}_{3}$ film are also provided in Supplementary Fig. 7. 
a
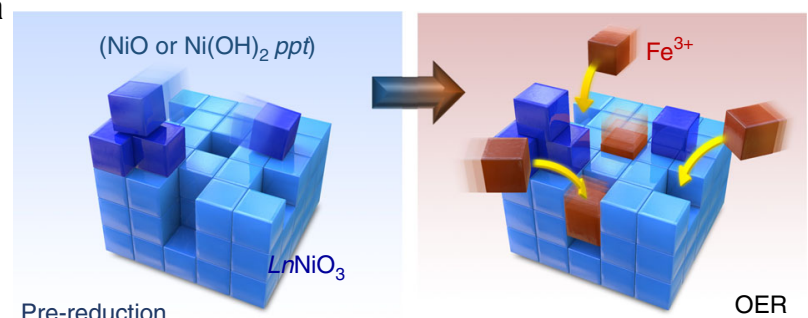

b

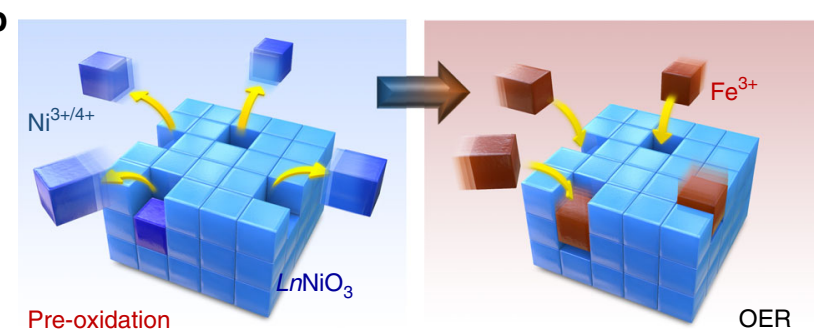

C Fe exchange via pre-oxidation

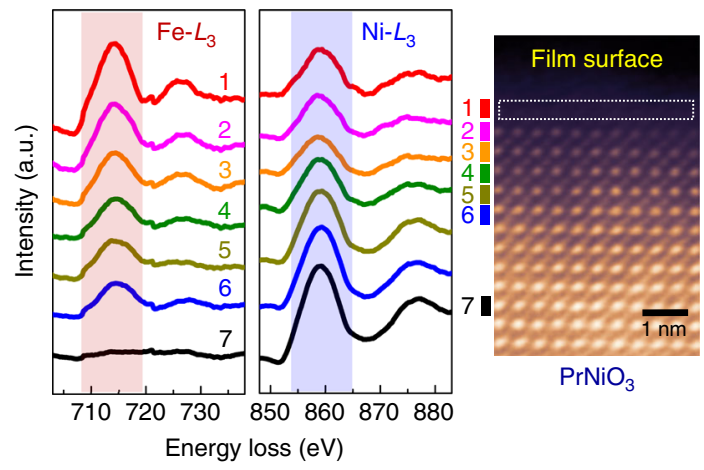

Fig. 2 Schematic illustrations and analytical evidence of surface Fe exchange. a Pre-reduction reactions are carried out in a $0.1 \mathrm{M} \mathrm{KOH}$ aqueous solution in a range of $0.62-1.3 \mathrm{~V}$ vs. reversible hydrogen electrode ( $\mathrm{RHE}$ ) so that $\mathrm{Ni}^{3+}$ in the nickelate lattice is extracted out. The resulting formation of nanoscale $\mathrm{NiO}$ (or $\mathrm{Ni}(\mathrm{OH})_{2}$ ) precipitates is identified. When the film after pre-reduction is oxidized in a 0.1-mM-Fe-containing $\mathrm{KOH}$ aqueous solution for measurement of the current density, $\mathrm{Fe}^{3+}$ ions in the solution appear to fill the Ni vacancies, resulting in the Fe exchange. $\mathbf{b} \mathrm{A}$ similar procedure occurs during the Fe exchange following the preoxidation reaction. $\mathrm{Ni}$ dissolution is observed when nickelate thin films are in highly oxidizing conditions ( $>1.75 \mathrm{~V}$ vs. RHE). Subsequently, the $\mathrm{Ni}$ vacancies created by the pre-oxidation reactions are filled again with $\mathrm{Fe}$ ions from the Fe-containing electrolyte during the oxygen evolution reaction (OER). c A layer-by-layer EELS analysis of $\mathrm{PrNiO}_{3}$ film subject to the Fe exchange via pre-oxidation demonstrates the gradual decline in the $\mathrm{Fe}-L_{3}$ peak intensity and simultaneous increase of the $\mathrm{Ni}-L_{3}$ peak, directly indicating that $\mathrm{Ni}$ is substituted by $\mathrm{Fe}$

Comparison of OER activities and electrochemical impedances. The plots in Fig. 3a compare the OER current densities of the different types of thin-film $\mathrm{LaNiO}_{3}$ samples, which are pristine (gray), 5\%-Fe-doped (black), and Fe-surface-exchange (blue and red). Five percent $\mathrm{Fe}$ was selected for the Fe-doped thin-film because this level of doping was observed to show the highest OER activity among the Fe-doped $\mathrm{LaNiO}_{3}$ films (see Supplementary Fig. 8 for details). The most important feature readily recognized in Fig. $3 \mathrm{a}$ is the exceptionally high OER current densities for the samples with the surface Fe exchange. In particular, as is apparent in the specific comparison in the bar graph in the right-hand column, the OER catalytic activity of the
Fe-exchange samples is nearly an order of magnitude higher than that of the Fe-doped solid-solution film. This strongly implies that there is another crucial contribution in addition to the $\mathrm{Fe}$ addition. To compare the OER activities of other Fe-containing perovskite oxides with those of our thin-film samples, we also provide an additional bar graph in Supplementary Fig. 9 based on the results of previous reports.

This same significantly higher activity following the ion exchange was also consistently found in both the $\mathrm{PrNiO}_{3}$ and $\mathrm{NdNiO}_{3}$ epitaxial films, as demonstrated in Fig. 3b, c, respectively. Therefore, the electrochemical Fe exchange appears to be a very effective way of achieving high OER activity in nickelate perovskites in general. As denoted by an arrow in the middle panel in Fig. 3a, the appearance of a small $\mathrm{Ni}^{2+} / \mathrm{Ni}^{3+}$ anodic peak between 1.45 and $1.5 \mathrm{~V}$ in the pre-reduction sample confirms the presence of $\mathrm{NiO}$ on the surface. This is in good agreement with the schematic illustration shown in Fig. 2a and the EDS results in Supplementary Fig. 3b (see Supplementary Fig. 10 for verification of the anodic peak in other pre-reduction nickelate films). The Tafel plots as well as the values of the Tafel slope for each nickelate are also provided in Supplementary Fig. 11.

Electrochemical impedance spectroscopy (EIS) was carried out to examine the electrical charge transfer behavior between the thin films and electrolyte solutions. Figure 4 shows three sets of Nyquist plots obtained by EIS for the $\mathrm{LaNiO}_{3}, \mathrm{PrNiO}_{3}$, and $\mathrm{NdNiO}_{3}$ thin films. As these nickelates are metallic ${ }^{43}$, the diameter of the first semicircle in the high-frequency range, which corresponds to the uncompensated resistance $\left(R_{\mathrm{u}}\right)$ between the working electrode and the reference electrode ${ }^{44}$, is very short in all three nickelates, showing $R_{\mathrm{u}}$ merely at an $\mathrm{ohm} \cdot \mathrm{cm}^{2}$ scale, as demonstrated in each of the insets (see Supplementary Fig. 12 for more details on the high-frequency arcs). Moreover, the $R_{\mathrm{u}}$ does not significantly vary, irrespective of whether the surface ion exchange has been conducted. In stark contrast, the thin-film samples following the Fe exchange show a huge difference in the diameter of the low-frequency second semicircle representing the resistance of charge transfer $\left(R_{\mathrm{CT}}\right)$ at the interface between the film and the electrolyte ${ }^{23}$. As clearly revealed in each Nyquist plot, this remarkable reduction in $R_{\mathrm{CT}}$ is observed for all the Fe-exchange samples. This notable decrease in charge-transfer barrier at the interface also agrees very well with the exceptionally high OER activity of the thin films following the surface Fe exchange. While there is no $R_{\mathrm{u}}$ variation, a substantially lower $R_{\mathrm{CT}}$ with increasing overpotential $(\eta)$ was also observed in each sample (see Supplementary Fig. 13), resulting in a Butler-Volmer type exponential increase in the OER current density as a function of $\eta$.

Direct observations with STEM. The added Fe clearly has a beneficial influence on the OER catalysis. However, the OER current density of the surface Fe-exchange $\mathrm{LaNiO}_{3}$ films was much higher than that of the Fe solid-solution film. This implies that the structural variation that occurs during the Fe exchange reactions may significantly enhance the catalytic activity. Investigating this using STEM at atomic resolution, we observed the surface region of the $\mathrm{LaNiO}_{3}$ films after pre-oxidation and prereduction. A striking structural feature was identified during the STEM observation: the oxygen anions were seriously displaced from their original positions, resulting in a remarkable distortion of the oxygen octahedra. Figure 5 a provides a representative example of the distorted octahedra captured by ABF imaging near the surface of a $\mathrm{LaNiO}_{3}$ film after eight cycles of pre-oxidation treatment. A series of magnified ABF images (lower panel) for five unit cells, denoted by yellow squares in the low-magnification 

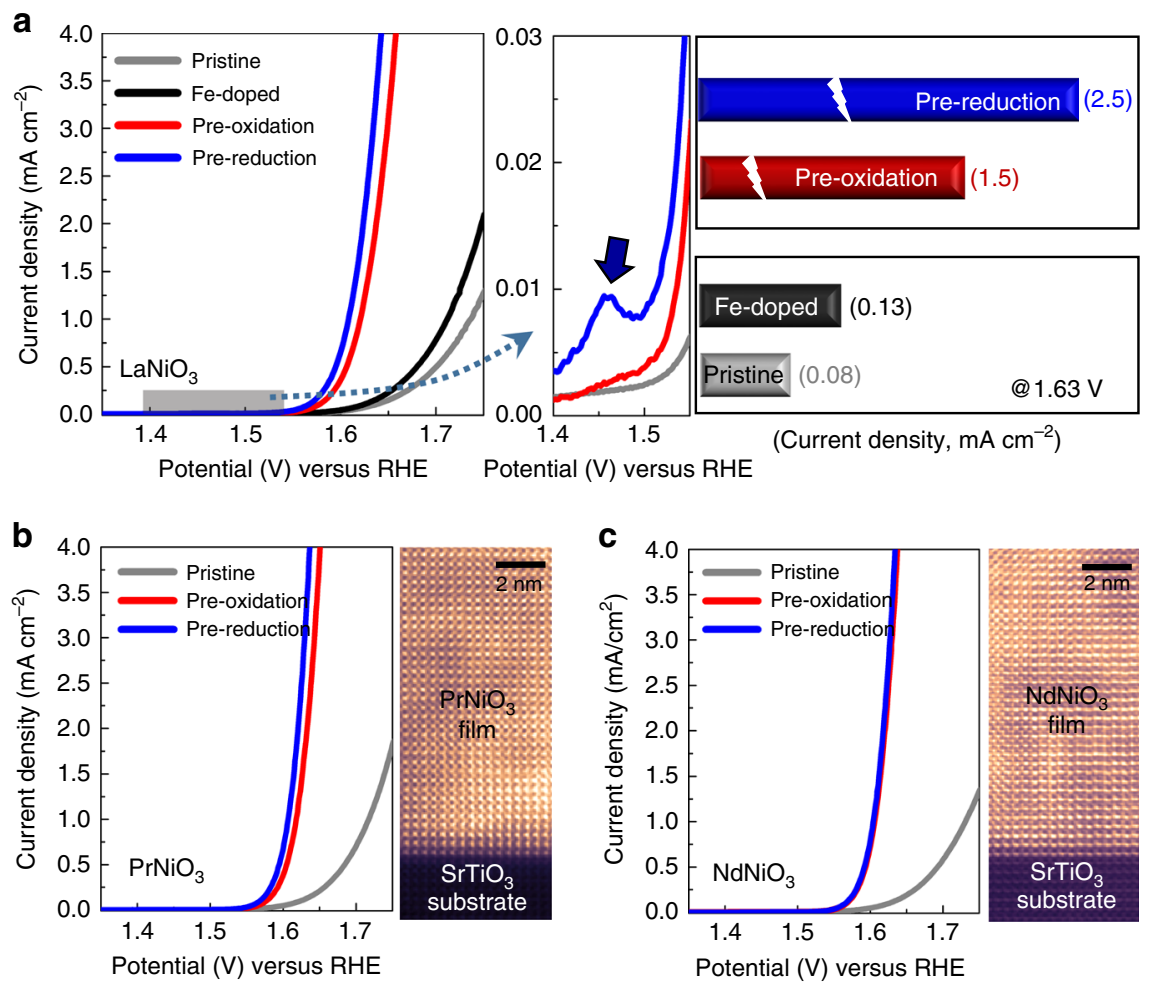

Fig. 3 Comparison of OER current densities. a Remarkably high OER activities were obtained in the $\mathrm{LaNiO}_{3}$ films following the surface Fe exchange that occurred with the pre-reduction and pre-oxidation reactions. Note the much larger OER current values of the surface-Fe-exchange films compared with the current value of the $\mathrm{La}(\mathrm{Ni}, \mathrm{Fe})_{3}$ solid-solution film, as specifically compared in the bar graph. The small peak indicated by a black arrow in the blue curve in the middle column represents the occurrence of the $\mathrm{Ni}^{2+} / \mathrm{Ni}^{3+}$ oxidation reaction. $\mathbf{b}, \mathbf{c}$ Remarkably enhanced OER activities were consistently identified in both $\mathrm{PrNiO}_{3}$ and $\mathrm{NdNiO}_{3}$ films following the surface Fe exchange. HAADF images also demonstrate the epitaxial growth of the films along with high crystallinity
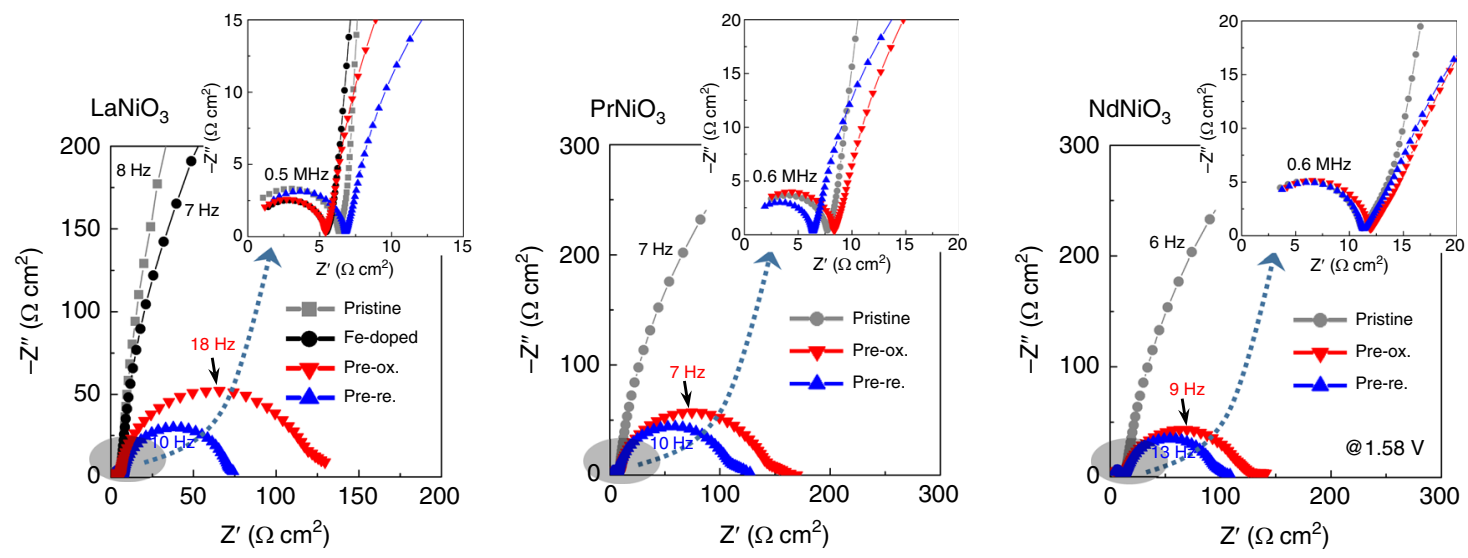

Fig. 4 Electrochemical impedance spectroscopy. In contrast to the low-frequency arcs of pristine films, considerably small arcs with very short diameters are noted for the surface-Fe-exchange films, and as a common feature in all three nickelates, indicating remarkably low charge-transfer resistance ( $R_{\mathrm{CT}}$ ) between the films and electrolytes. As can be seen in each magnified inset for the high-frequency range, denoted by a gray shadow, the electrolyte resistance $\left(R_{\mathrm{E}}\right)$ values hardly differ between the samples in each of the nickelates

image, were compared with the unit-cell image showing four oxygens placed immediately at the corners of a white rhombus for a Ni octahedron in the bulk (upper right panel). The comparison directly shows that most oxygens have been considerably displaced from their original positions in the bulk, as indicated by a distorted yellow rhombus in each of the images (see Supplementary Fig. 14 for the unfiltered raw images). Altogether with the ABF image obtained from the bulk film in Supplementary Fig. 15, an additional set of ABF images for the surface region from the pristine sample without pre-oxidation or pre-reduction is provided in Supplementary Fig. 16 to confirm the undistorted oxygen octahedra as a standard.

ABF-STEM is one of the imaging techniques commonly used for visualizing the position of light elements including oxygen ${ }^{20,45-50}$. However, because atomic columns are imaged in this STEM mode using a dark contrast with a bright background, the position uncertainty of light elements is sometimes not negligible when a specimen is comparatively thick and its tilting angle is high $^{51,52}$. Therefore, to achieve a better signal-to-noise ratio, we used an imaging method, iDPC-STEM, where four-quadrant 
a
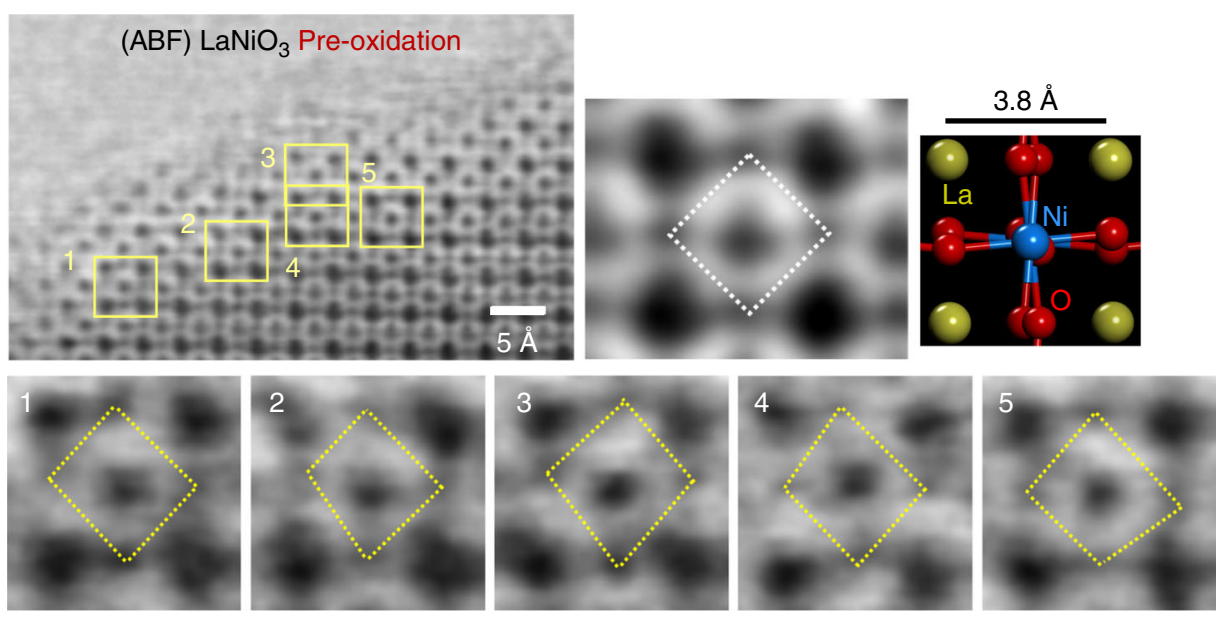

b
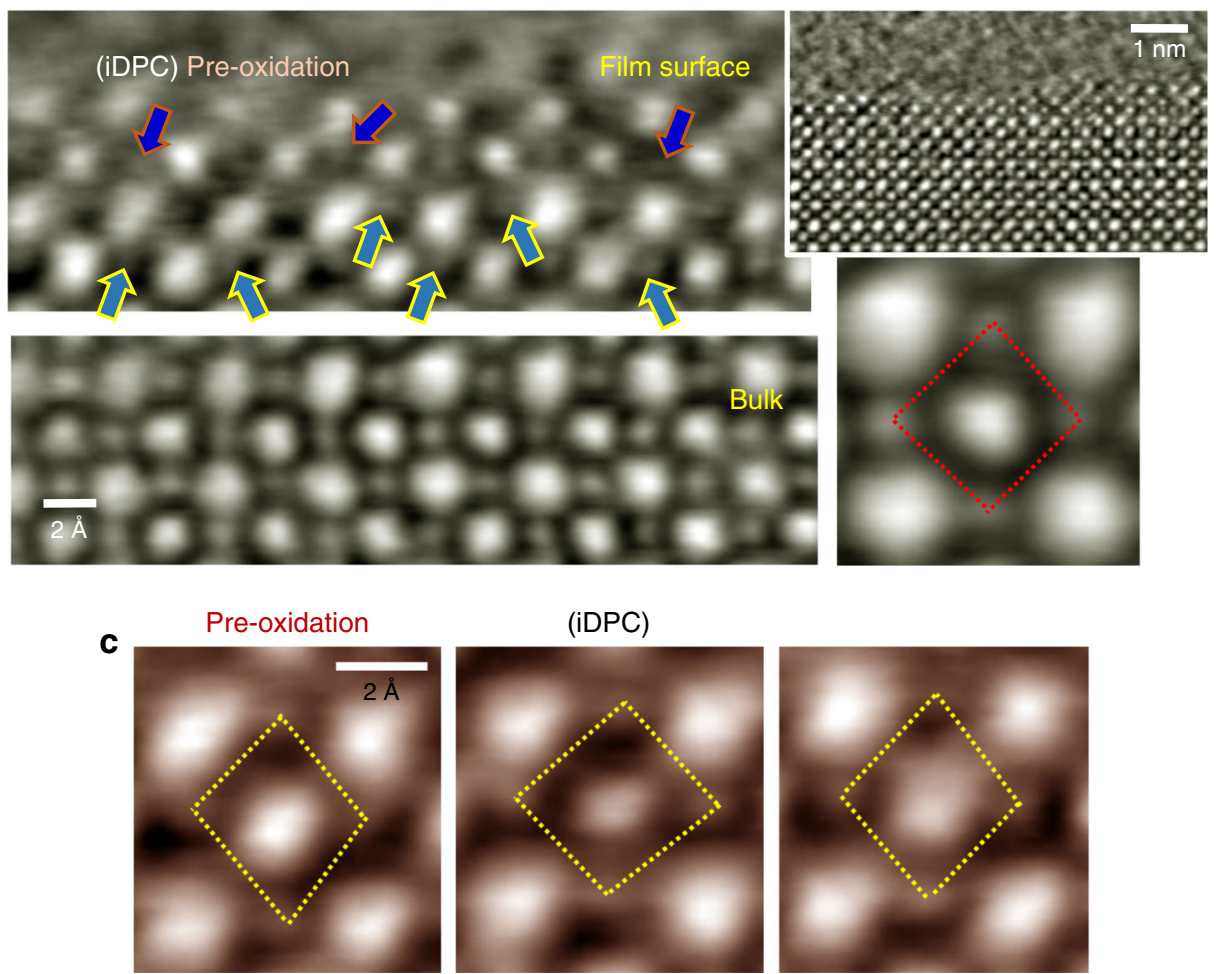

Pre-reduction
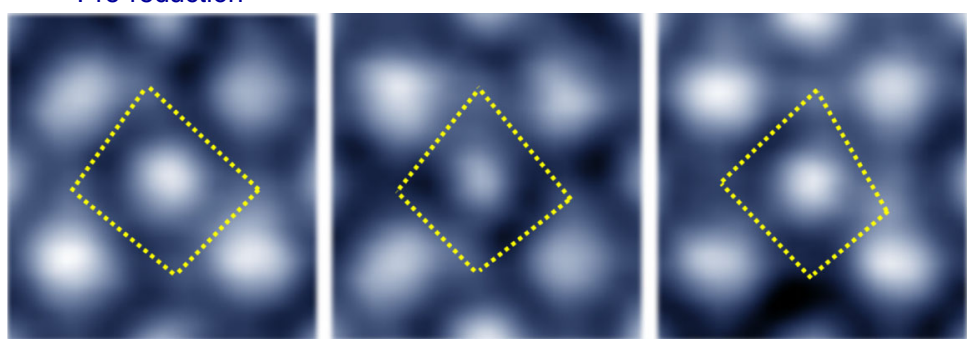

Fig. 5 Atomic-scale direct evidence of oxygen-octahedron distortion. The surface region of the $\mathrm{LaNiO}_{3}$ films after pre-oxidation and pre-reduction was observed in scanning transmission electron microscopy (STEM). a The unit-cell image with the indication of a white diamond was acquired from the bulk region for reference. A series of annular bright-field (ABF) STEM images are provided in the lower panel for the unit cells denoted by a yellow square in the low-magnification image. As indicated by a yellow rhombus, each oxygen octahedron is substantially distorted. $\mathbf{b}$ Integrated differential phase-contrast (iDPC) STEM shows consistent image features. As indicated by arrows on the image captured from the film surface, serious displacement of oxygen atoms is clarified. c iDPC images exemplifying the oxygen displacement are demonstrated for both pre-oxidation and pre-reduction cases 
a
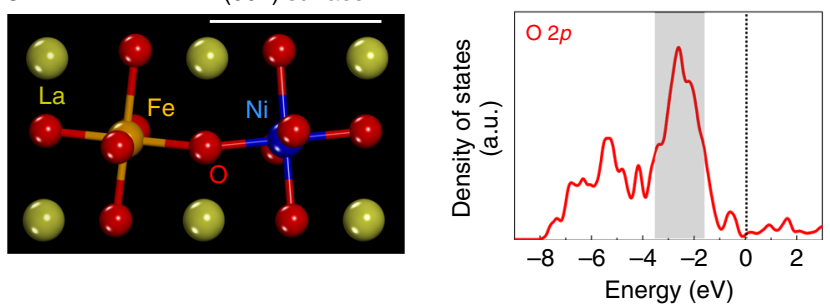

b
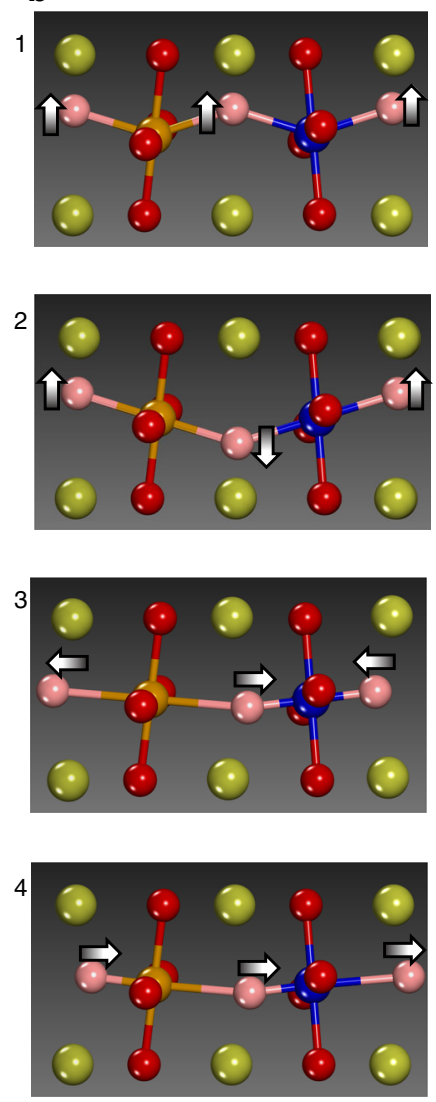
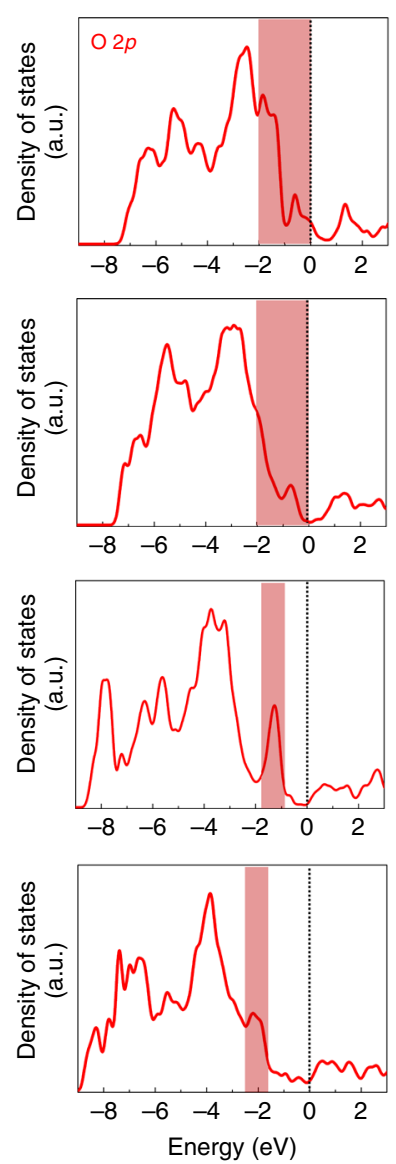
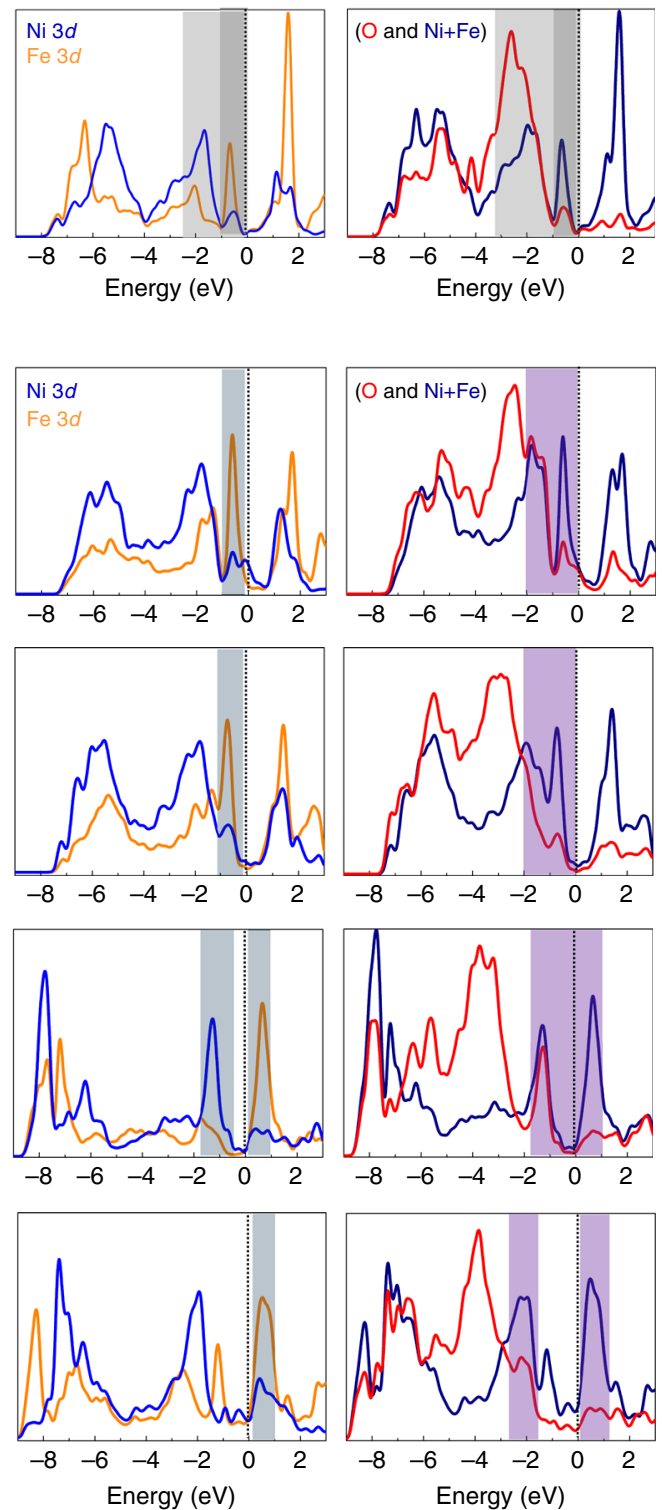

Fig. 6 Density of states (DOS) of (001) $\mathrm{LaNiO}_{3}$ with $\mathrm{Fe}$. A set of DOS plots for $\mathrm{O} 2 p, \mathrm{Ni} / \mathrm{Fe} 3 d$, and metal/oxygen total states are provided for each case. a An optimized supercell of the (001) surface and its DOS are taken as references for comparison. A relatively high density of states in each DOS plot is denoted by a gray shadow. Note that fairly high Fe-3d states are identified between -1 and 0 eV below the Fermi level. b Four independent cases for oxygen-octahedron distortion are included, showing vertical (cases 1 and 2) and lateral (cases 3 and 4) displacements of oxygen. The significant variations in DOS produced by the oxygen displacements are notable features in the electronic structure. In particular, the substantial increase in the DOS between -2 and $+1 \mathrm{eV}$ near the Fermi level has a beneficial effect permitting easier charge transfer between oxygen and metals

segmented STEM detectors are utilized for differential phasecontrast image acquisition ${ }^{30,31}$ (see Supplementary Fig. 17 for more details on iDPC-STEM). Figure 5b shows typical iDPC images taken of $\mathrm{LaNiO}_{3}$ thin-film samples after the pre-oxidation reactions. In contrast to the well-ordered pseudocubic array of oxygens in the bulk, denoted by a red diamond in the unit-cell image, the arrows in the iDPC image of the surface region directly indicate considerable displacements of oxygen anions. Two series of enlargements along with the indication by yellow rhombuses shown in Fig. $5 \mathrm{c}$ provide compelling evidence of the serious distortion of the oxygen octahedra. Based on these two independent STEM imaging methods, it appears that the $\mathrm{Ni}$ extraction is inevitably accompanied by perturbation of the oxygen-anion framework during the pre-oxidation and prereduction reactions, resulting in serious displacement of oxygens.
It is noted that oxygen distortion, in fact, should not be negligible along the $z$-axis, although the images in Fig. $5 \mathrm{~b}$ merely demonstrate two-dimensional distortions in the $x-y$ plane. As strong $z$-axis distortion indicates that the displaced oxygen is no longer on the focal plane during the STEM image acquisition, substantially weak column intensity cannot be avoided. As a consequence, it is very likely that $z$-axis distortion of oxygen is responsible for the low intensity of some oxygen columns, for example, in the last image in Fig. 5c.

DFT calculations. Since the first publication of the Jahn-Teller theorem in 1937 (ref. ${ }^{53}$ ), it has been widely understood that structural perturbation in transition-metal compounds strongly correlates with changes in the energy state of electrons. Therefore, 
we carried out ab initio DFT calculations to examine how the density of states (DOS) of the O $2 p$ and Ni/Fe $3 d$ orbitals in the (001) $\mathrm{LaNiO}_{3}$ surface would be affected by the oxygenoctahedron distortion. The atomic illustration shown in Fig. 6a presents part of the (001) surface layer in an Fe-doped $\mathrm{LaNiO}_{3}$ supercell after geometric optimization. As denoted by the dark gray shadow in the Ni/Fe DOS in Fig. 6a, one noteworthy aspect regarding the $\mathrm{Fe}$ addition to $\mathrm{LaNiO}_{3}$ is that the $\mathrm{Fe} 3 d$ states make a substantial contribution to the DOS in the range from -1 to $0 \mathrm{eV}$, where the number of DOS in the pristine $\mathrm{LaNiO}_{3}$ surface is significantly small (see Supplementary Fig. 18a for a set of the DOS plots of the pristine (001) surface without $\mathrm{Fe}$ ).

More importantly, we identified notable variations in the DOS of both the $\mathrm{O} 2 p$ and $\mathrm{Ni} / \mathrm{Fe} 3 d$ orbitals when a subangstrom scale of displacement was introduced in the oxygen anions in each of the supercells. Figure $6 \mathrm{~b}$ shows four different cases of oxygen displacements and corresponding DOS plots of $\mathrm{O} 2 p$ and $\mathrm{Ni} / \mathrm{Fe}$ $3 d$ levels, demonstrating vertical (cases 1 and 2) and lateral (cases 3 and 4) displacements of oxygens (light pink spheres) with neighboring $\mathrm{Ni}$ (blue sphere) and $\mathrm{Fe}$ (orange sphere). Specific values for each of the oxygen displacements are provided in Supplementary Fig. 19. Compared with the DOS of the (001) surface shown in Fig. 6a, several significant features can be recognized in these sets of DOS with the oxygen displacements. First, as denoted by the red and blue shadows in the $\mathrm{O} 2 p$ and $\mathrm{Ni} /$ Fe $3 d$ DOS plots, respectively, there is a considerable increase in electronic states in the range from -2 to $+1 \mathrm{eV}$. For example, a much higher density of the $\mathrm{O} 2 p$ states between -2 and $0 \mathrm{eV}$ is easily observed in cases 1 and 3 . A rise in the $\mathrm{Fe} 3 d$ states between -1 and $0 \mathrm{eV}$ caused by the vertical shift of oxygens (cases 1 and 2) is another feature that highlights the effect of oxygen displacements. Further, cases 3 and 4 demonstrate that the lateral shift of oxygens can lead to a substantial increase in the Fe $3 d$ states between 0 and $+1 \mathrm{eV}$ above the Fermi level, while also contributing to the increase in the $\mathrm{Ni} 3 d$ states between -2 and $-1 \mathrm{eV}$. This high density of Fe $3 d$ states near the Fermi level appears to be more dominant compared with the Ni-3d DOS variation induced by the oxygen displacements without $\mathrm{Fe}$, as shown in Supplementary Fig. 18b.

\section{Discussion}

Although various factors are involved in oxygen electrocatalysis performance, the charge transfer between transition metals and oxygen appears to be one of the more critical steps. Indeed, as rate-limiting reactions with large activation barriers are usually involved in the formation of intermediate adsorbates of $\mathrm{OH}^{*}$ and $\mathrm{O}^{\star}$ during the OER, facilitating the transfer of electrons between transition metals and adsorbates via oxygen is an important issue $18,25,54,55$. The charge transfer from transition metals to adsorbates via oxygen (e.g., $\mathrm{Ni}^{3+}{ }_{-} \mathrm{O}\left(\mathrm{OH}^{\star}\right)^{-} \rightarrow \mathrm{Ni}^{4+}{ }_{-} \mathrm{OO}^{\star 2-}$ ) would be much easier and more likely to occur if a larger number of density of $\mathrm{O} 2 p$ and metal $3 d$ bonding states were placed near the Fermi level rather than far below it. Consequently, as has already been suggested in previous reports, an $\mathrm{O} 2 \mathrm{p}$ band center with a higher position and a higher degree of covalency between metals and oxygen are both beneficial for the OER. Similarly, electron transfer from the $\mathrm{O} 2 p$ band to the empty metal $3 d$ band (e.g., $\mathrm{Ni}^{4+}{ }_{-} \mathrm{O}^{2-} \rightarrow \mathrm{Ni}^{3+}-\mathrm{O}\left(\mathrm{OH}^{*}\right)^{-}$) also can be remarkably facilitated if a large number of metal $3 d$ antibonding states are present as close as possible to the Fermi level, so that the charge transfer energy (the energy difference between the unoccupied $\mathrm{Ni}$ $3 d$ and occupied O $2 p$ band centers) is drastically reduced. In this regard, our DFT calculations for the series of DOS in Fig. 6 directly demonstrate that the distortion of oxygen octahedra is a very effective approach for improving charge transfer between transition metals and oxygen. These results are also in excellent agreement with the exceptional order-of-magnitude improvement in the OER activity of the Fe-exchange samples.

Although more rigorous and extensive investigations are required to precisely understand the changes in DOS induced by the distortion of octahedra, the Jahn-Teller theorem can be applied to qualitatively explain this notable change. Each of the degenerate $t_{2 \mathrm{~g}}\left(d_{\mathrm{xy}}, d_{\mathrm{yz}}\right.$, and $\left.d_{\mathrm{zx}}\right)$ and $e_{\mathrm{g}}\left(d_{\mathrm{x}}^{2}{ }^{2}{ }_{\mathrm{y}}^{2}\right.$ and $\left.d_{\mathrm{z}}{ }^{2}\right)$ orbitals discreetly splits under the cubic symmetry of oxygen ligands, constructing an equally degenerate upper $e_{\mathrm{g}}$ level and a triply degenerate lower $t_{2 g}$ level. However, when the ligands are strongly distorted, such degeneracy is significantly removed in each level, thereby resulting in the nondegenerate five $d$ orbitals with various splitting energy gaps. Therefore, the energy range of the bonding states below the Fermi level can be widened, as can be seen in cases 3 and 4 in Fig. 6b. At the same time, a higher DOS may be induced near the Fermi level as a consequence of the narrower gap between the bonding and antibonding states, as denoted by the blue shadows in the Ni/Fe $3 d$ DOS plots in Fig. 6 b.

Many previous studies on the OER catalysis of oxides have attempted to relate the observed variation in OER activity with the electronic structure of the bulk and the structural change of the overall polycrystals, even though there is substantial crystallographic anisotropy in the catalytic activity ${ }^{13,56}$, which strongly depends on both electronic and atomic structures near the surface. To move past these previous limitations, our study employed a combination of (001) epitaxial thin films, atomic-scale structure and composition analyses, and DFT calculations using more realistic supercells from direct observation. We were thus able to reliably demonstrate a solid correlation among the structural perturbation, the electronic structure, and the resultant OER property in nickelate perovskites. In addition to providing evidence that the $\mathrm{Fe}$ exchange has a rather general impact on the exceptional increase in OER activity in nickelate perovskites, the findings in the present work show that symmetry-breaking configurational control of atoms on the surface can offer an important platform toward exceptional oxygen electrocatalysis in perovskite oxides.

We have demonstrated that $\mathrm{Fe}$ exchange via selective $\mathrm{Ni}$ extraction in the surface region of (001) thin-film nickelate perovskites by electrochemical oxidation and reduction reactions is a remarkably efficient method of achieving exceptional OER activity. In particular, we probed the strong distortion of oxygen octahedra induced by the Ni extraction during the pre-oxidation and pre-reduction steps. A systematic series of DFT calculations showed that this atomic-scale structural perturbation resulted in a significant variation in the $\mathrm{O} 2 p$ and $\mathrm{Ni} / \mathrm{Fe} 3 d$ states, especially near the Fermi level, leading to much easier charge transfer between metals and oxygen. Our work highlights the impact of symmetry-broken oxygen geometry on electronic structure and the resulting oxygen electrocatalysis in perovskite oxides.

\section{Methods}

Thin-film fabrication. Epitaxial $L n \mathrm{NiO}_{3}(L n=\mathrm{La}, \mathrm{Pr}, \mathrm{Nd})$ thin films were fabricated by using a sol-gel process. First, $\mathrm{La}\left(\mathrm{NO}_{3}\right)_{3} \cdot 6 \mathrm{H}_{2} \mathrm{O}(99.999 \%$, Aldrich), $\mathrm{Pr}$ $\left(\mathrm{NO}_{3}\right)_{3} \cdot 6 \mathrm{H}_{2} \mathrm{O}\left(99.99 \%\right.$, Aldrich), $\mathrm{Nd}\left(\mathrm{NO}_{3}\right)_{3} \cdot 6 \mathrm{H}_{2} \mathrm{O}$ (99.9\%, Aldrich), and $\mathrm{Ni}$ $\left(\mathrm{CH}_{3} \mathrm{COO}\right)_{2} \cdot 4 \mathrm{H}_{2} \mathrm{O}(99.998 \%$, Aldrich) were used as starting materials for the preparation of precursor solutions. Each of the lanthanide nitrates and the nicke acetate were first dissolved in 2-methoxyethanol under a constant stirring condition to prepare precursor solutions with $0.2 \mathrm{M}$. For complete dissolution of the source materials, refluxing was carried out at $80^{\circ} \mathrm{C}$ for $1 \mathrm{~h}$. Each of the precursor solutions was deposited on (001) $\mathrm{SrTiO}_{3}$ single-crystal substrates by a spin-coating method at $5000 \mathrm{rpm}$ for $10 \mathrm{~s}$. The wet films were dried at $150^{\circ} \mathrm{C}$ for $10 \mathrm{~min}$ on a hot plate, subsequently heat-treated at $400^{\circ} \mathrm{C}$ for $10 \mathrm{~min}$ for pyrolysis, and finally annealed at $800^{\circ} \mathrm{C}$ for $1 \mathrm{~h}$ in air for $\mathrm{LaNiO}_{3}$ films, and at $700^{\circ} \mathrm{C}$ for $30 \mathrm{~min}$ in an $\mathrm{O}_{2}$-flow $\left(400 \mathrm{sccm}\right.$ ) atmosphere for $\mathrm{PrNiO}_{3}$ and $\mathrm{NdNiO}_{3}$ films for crystallization. The epitaxy of the grown films was confirmed by X-ray diffractometry (X'PertPRO MRD, PANalytical) with $\mathrm{Cu}-K_{\alpha}$ radiation and STEM observation. 
Scanning transmission electron microscopy, energy-dispersive X-ray spectroscopy, and electron energy-loss spectroscopy. Samples for STEM observation were prepared by lift out via ion-beam milling in a focused ion-beam system (Quanta 3D FEG and Helios G4 UX, Thermo Fisher Scientific). Protective amorphous carbon and thin Pt layers were applied over the region of interest before milling. To minimize the sidewall damage and sufficiently thin the specimen for electron transparency, final milling was carried out at a voltage of $\sim 2 \mathrm{kV}$. Conventional STEM images were taken with a transmission electron microscope (Titan cubed G2 60-300, Thermo Fisher Scientific) at $300 \mathrm{kV}$ with a spherical aberration (Cs) corrector (CEOS GmbH). The optimum size of the electron probe was $\sim 1 \AA$ with a convergence semiangle of $19 \mathrm{mrad}$. The collection semiangles of the STEM detectors were set to 67.6-200 mrad for HAADF imaging and 12.1-67.6 mrad for $\mathrm{ABF}$ imaging. iDPC images were obtained using a four-quadrant segmented detector attached in Titan Themis Z (Thermo Fisher Scientific) with a Cs corrector at $300 \mathrm{kV}$. The obtained raw images were band-pass filtered to reduce background noise. Chemical mapping with EDS was carried out in the Titan cubed G2 at 300 $\mathrm{kV}$ along with four integrated silicon-drift EDS detectors (ChemiSTEM ${ }^{\mathrm{rx}}$ technology) at a collection solid angle of 0.7 srad. La- $L_{\alpha}(4.6 \mathrm{keV}), \operatorname{Pr}-L_{\alpha}(5.0 \mathrm{keV}), \mathrm{Nd}-$ $L_{\alpha}(5.2 \mathrm{keV}), \mathrm{Ni}-K_{\alpha}(7.5 \mathrm{keV})$, and $\mathrm{Fe}-K_{\alpha}(6.4 \mathrm{keV})$ lines were selected during elemental mapping. The probe current was adjusted to be $50-100 \mathrm{pA}$ with a scanning time of $<300 \mathrm{~s}$. The EDS maps were low-pass filtered using Bruker ESPRIT software after the reduction of background noise for better visualization. EELS analysis was performed with a Gatan Image Filter (GIF Quantum 965, Gatan Inc.). Electron energy-loss spectra for the $\mathrm{Ni}-L$ and Fe- $L$ edges were acquired for spectrum imaging with a dispersion of $0.25 \mathrm{eV}$ per channel and a collection aperture of $5 \mathrm{~mm}$ in diameter.

Electrochemical characterization. All electrochemical reactions and measure ments were conducted with a potentiostat (Biologic SP-300) in a $0.1 \mathrm{M} \mathrm{KOH}$ aqueous solution $(\mathrm{pH}=12.9)$ prepared by using the Milli-Q water $(18.2 \mathrm{M} \Omega \mathrm{cm})$ and $\mathrm{KOH}$ pellets (Sigma Aldrich, $99.99 \%$ ) to achieve sufficiently high purity. According to the chemical analysis by inductively coupled plasma-optical emission spectrometry (ICP-OES), the Fe impurity in our electrolyte solutions was identified to be less than $5 \mathrm{ppb}$. Furthermore, when we carried out the oxidation reactions up to $1.85 \mathrm{~V}$ several times, we found that the OER current density continuously decreased (see Supplementary Fig. 20), directly demonstrating the absence of an Fe-impurity effect in the electrolyte. A Pt counter electrode and a saturated $\mathrm{Ag} / \mathrm{AgCl}$ reference electrode were used. The measured potential values vs. the $\mathrm{Ag} / \mathrm{AgCl}$ reference electrode were converted into the RHE scale by using the following equation at $25^{\circ} \mathrm{C}$,

$$
E_{\mathrm{RHE}}=E_{\mathrm{Ag} / \mathrm{AgCl}}+0.059 \cdot \mathrm{pH}+E_{\mathrm{Ag} / \mathrm{AgCl}}^{\circ}
$$

where $E_{\mathrm{RHE}}$ is the converted potential vs. RHE, $E_{\mathrm{Ag} / \mathrm{AgCl}}$ is the measured potential against the $\mathrm{Ag} / \mathrm{AgCl}$ reference electrode, and $E_{\mathrm{Ag} / \mathrm{AgCl}}^{\circ}$ is the standard potential of $\mathrm{Ag} / \mathrm{AgCl}(\mathrm{KCl} 3 \mathrm{M})$ at $25^{\circ} \mathrm{C}$, i.e., $0.21 \mathrm{~V}$. Pre-reduction reactions (up to five cycles) were carried out in a $0.1 \mathrm{M} \mathrm{KOH}$ aqueous solution in a potential range from 1.30 down to $0.62 \mathrm{~V}$ vs. $\mathrm{RHE}$, which belongs to the stable range of $\mathrm{Ni}^{2+}$ (approximately $0.1-1.3 \mathrm{~V}$ vs. $\mathrm{RHE}$ at $\mathrm{pH}=12.9)^{57}$, at a sweeping rate of $10 \mathrm{mV} / \mathrm{s}$. Pre-oxidation reactions (up to six cycles) were also conducted in an identical manner in a potential range from 1.27 to $1.85 \mathrm{~V}$ vs. RHE. For Fe exchange after the prereduction or pre-oxidation reactions and simultaneously cyclic potential was applied (ten cycles) to the samples in a range from 1.27 to $1.75 \mathrm{~V}$ vs. RHE in a 0.1 $\mathrm{M} \mathrm{KOH}$ aqueous solution with 0.1 (or 1.0) $\mathrm{mM} \mathrm{Fe}\left(\mathrm{NO}_{3}\right)_{3} \cdot 9 \mathrm{H}_{2} \mathrm{O}$ so that $\mathrm{Ni}$ vacancies created in the surface region of the films could be filled with Fe from the solution (see Supplementary Fig. 21 for the potential range comparison between the pre-reduction, pre-oxidation, and Fe exchange reactions). OER current densities were also simultaneously reordered in the same Fe-containing $\mathrm{KOH}$ solution. As reference data for comparison, the OER current densities of pristine and $\mathrm{Fe}$ doped thin-film samples were also measured in the same potential range (1.27-1.75 V vs. RHE) in a Fe-free $\mathrm{KOH}$ solution. All electrolyte solutions were presaturated by bubbling $\mathrm{O}_{2}$ for 30 min under constant $\mathrm{O}_{2}$ bubbling. The substrate and the connecting copper wire were completely covered with chemically inert insulating epoxy resin after application of silver paint on the back side of a thinfilm sample so as to expose the film surface only. Electrochemical impedance spectroscopy to investigate both the uncompensated series resistance $\left(R_{\mathrm{u}}\right)$ for $i R_{\mathrm{u}}$ correction of the applied potential and the interface charge-transfer resistance was also carried out in the same potentiostat in a frequency range from $0.1 \mathrm{~Hz}$ to 1 $\mathrm{MHz}$ with an amplitude of $10 \mathrm{mV}$. Double layer capacitance was measured to examine the change in comparative surface roughness after the $\mathrm{Fe}$ exchange. As previously reported ${ }^{58}$, the capacitance measurements were carried out at $0.05-0.20$ $\mathrm{V}$ vs. Ag/AgCl. No significant variation in capacitance was identified in each nickelate case, verifying that there was no change in the surface morphology responsible for the exceptional increase of OER activity.

DFT calculations. Ab initio DFT calculations for DOS variation at the (001) surface of pristine and Fe-doped $\mathrm{LaNiO}_{3}$ were carried out using the spin-polarized local density approximation (LDA) functional for exchange correlation, along with the ultrasoft pseudopotentials for ionic cores, as implemented in the CASTEP code (Biovia Inc.). A sufficiently long (001)-surface slab along with a 10 - $\AA$ vacuum layer was constructed as an optimum supercell for each calculation to make the relaxation layer of each slab more than $10 \AA$ in thickness. To account for the electron localization around $\mathrm{Ni}$ and Fe ions, the LDA $+U$ method with the Hubbard $U$ parameter ( $4.0 \mathrm{eV}$ for both $\mathrm{Ni} 3 d$ and $\mathrm{Fe} 3 d$ states) was employed ${ }^{59,60}$ Low-spin $\left(t_{2 g}{ }^{6}\right)\left(e_{g}{ }^{1}\right)$ for $d^{7} \mathrm{Ni}^{3+}$ and high-spin $\left(t_{2 g}{ }^{3}\right)\left(e_{g}^{2}\right)$ for $d^{5} \mathrm{Fe}^{3+}$ configurations were assumed, respectively ${ }^{61}$. The plane-wave basis set for the kinetic energy cutoff was $500 \mathrm{eV}$. Relaxation of the internal coordinates for each atom was performed using the Broyden-Fletcher-Goldfarb-Shanno (BFGS) algorithm with convergence tolerances of $0.1 \mathrm{eV} / \AA$ for the maximum ionic force, $5 \times 10^{-5} \mathrm{eV} /$ atom for the total energy, and $0.005 \AA$ for the maximum ionic displacement. The charge variation of each oxygen atom before and after the formation of $\mathrm{Ni}$ vacancy could be obtained from the Mulliken electronic population analysis 62,63

\section{Data availability}

The data that support the findings of this study are available from the corresponding author (S.-Y.C.) upon reasonable request.

Received: 3 January 2019 Accepted: 4 June 2019

Published online: 20 June 2019

\section{References}

1. Katsounaros, I., Cherevko, S., Zeradjanin, A. R. \& Mayrhofer, K. J. J. Oxygen electrochemistry as a cornerstone for sustainable energy conversion. Angew. Chem. Int. Ed. 53, 102-121 (2014).

2. Fabbri, E., Habereder, A., Waltar, K., Kötz, R. \& Schmidt, T. J. Development and perspectives of oxide-based catalysts for the oxygen evolution reaction. Catal. Sci. Technol. 4, 3800-3821 (2014).

3. Chen, D., Chen, C., Baiyee, Z. M., Shao, Z. \& Ciucci, F. Nonstoichiometric oxides as low-cost highly-efficient oxygen reduction/evolution catalysts for low-temperature electrochemical devices. Chem. Rev. 115, 9869-9921 (2015).

4. Roger, I., Shipman, M. A. \& Symes, M. D. Earth-abundant catalysts for electrochemical and photoelectrochemical water splitting. Nat. Rev. Chem. 1, 3 (2017).

5. Song, F. et al. Transition metals oxides as electrocatalysts for the oxygen evolution reaction in alkaline solution: an application-inspired renaissance. $J$. Am. Chem. Soc. 140, 7748-7759 (2018).

6. Bockris, J. O. 'M. \& Otagawa, T. Mechanism of oxygen evolution on perovskites. J. Chem. Phys. 87, 2960-2971 (1983).

7. Bockris, J. O. 'M. \& Otagawa, T. The electrocatalysis of oxygen evolution on perovskites. J. Electrochem. Soc. 131, 290-302 (1984).

8. Zhu, H., Zhang, P. \& Dai, S. Recent advances of lanthanum-based perovskite oxides for catalysis. ACS Catal. 5, 6370-6385 (2015).

9. Cheng, X. et al. Oxygen evolution reaction on perovskites: A multieffect descriptor study combining experimental and theoretical methods. ACS Catal. 8, 9567-9578 (2018).

10. Suntivich, J., May, K. J., Gasteiger, H. A., Goodenough, J. B. \& Shao-Horn, Y A perovskite oxide optimized for oxygen evolution catalysis from molecular orbital principles. Science 334, 1383-1385 (2011).

11. Grimaud, A. et al. Double perovskites as a family of highly active catalysts for oxygen evolution in alkaline solution. Nat. Commun. 4, 2439 (2013).

12. Kim, J., Yin, X., Tsao, K. -C., Fang, S. \& Yang, H. $\mathrm{Ca}_{2} \mathrm{Mn}_{2} \mathrm{O}_{5}$ as oxygendeficient perovskite electrocatalyst for oxygen evolution reaction. J. Am. Chem. Soc. 136, 14646-14649 (2014).

13. Chang, S. H. et al. Functional links between stability and reactivity of strontium ruthanate single crystals during oxygen evolution. Nat. Commun. 5, 4191 (2014).

14. Yagi, S. et al. Covalency-reinforced oxygen evolution reaction catalyst. Nat. Commun. 6, 8249 (2015).

15. Mefford, J. T. et al. Water electrolysis on $\mathrm{La}_{1-}{ }_{x} \mathrm{Sr}_{x} \mathrm{CoO}_{3^{-}} \delta$ perovskite electrocatalysts. Nat. Commun. 7, 11053 (2016)

16. Liu, H. et al. Promoting photochemical water oxidation with metallic band structures. J. Am. Chem. Soc. 138, 1527-1535 (2016).

17. Seitz, L. C. et al. A highly active and stable $\mathrm{IrO}_{2} / \mathrm{SrIrO}_{3}$ catalyst for the oxygen evolution reaction. Science 353, 1011-1014 (2016).

18. Hong, W. T. et al. Charge-transfer-energy-dependent oxygen evolution reaction mechanisms for perovskite oxides. Energy Environ. Sci. 10, 2190-2200 (2017)

19. Grimaud, A. et al. Activating lattice oxygen redox reactions in meal oxides to catalyse oxygen evolution. Nat. Chem. 9, 457-465 (2017).

20. Bak, J., Bae, H. B., Kim, J., Oh, J. \& Chung, S. -Y. Formation of twodimensional homologous faults and oxygen electrocatalytic activities in a perovskite nickelate. Nano Lett. 17, 3126-3132 (2017).

21. Man, I. C. et al. Universality in oxygen evolution electrocatalysis on oxide surfaces. ChemCatChem 3, 1159-1165 (2011).

22. Subbaraman, R. et al. Trends in activity for the water electrolyser reactions on $3 d \mathrm{M}(\mathrm{Ni}, \mathrm{Co}, \mathrm{Fe}, \mathrm{Mn})$ hydr(oxy)oxide catalysts. Nat. Mater. 11, 550-557 (2012). 
23. Morales-Guio, C. G., Liardet, L. \& Hu, X. Oxidatively electrodeposited thinfilm transient metal (oxy)hydroxides as oxygen evolution catalysts. J. Am. Chem. Soc. 138, 8946-8957 (2016).

24. Zhou, Y. et al. Enlarged Co-O covalency in octahedral sites leading to highly efficient spinel oxides for oxygen evolution reaction. Adv. Mater. 30, 1902912 (2018).

25. Heo, Y. et al. Symmetry-broken atom configurations at grain boundaries and oxygen evolution electrocatalysis in perovskite oxides. Adv. Energy Mater. 8, 1802481 (2018).

26. Petrie, J. R. et al. Enhanced bifunctional oxygen catalysis in strained $\mathrm{LaNiO}_{3}$ perovskites. J. Am. Chem. Soc. 138, 2488-2491 (2016).

27. Ek, M., Ramasse, Q. M., Arnarson, L., Moses, P. G. \& Helveg, S. Visualizing atomic-scale redox dynamics in vanadium oxide-based catalysts. Nat. Commun. 8, 305 (2017).

28. Li, T. et al. Atomic-scale insights into surface species of electrocatalysts in three dimension. Nat. Catal. 1, 300-305 (2018).

29. Wang, $Z$. et al. Overall water splitting by $\mathrm{Ta}_{3} \mathrm{~N}_{5}$ nanorod single crystals grown on the edges of $\mathrm{KTaO}_{3}$ particles. Nat. Catal. 1, 756-763 (2018).

30. Lazić, I., Bosch, E. G. T. \& Lazar, S. Phase contrast STEM for thin samples: Integrated differential phase contrast. Ultramicroscopy 160, 265-280 (2016).

31. Yücelen, E., Lazić, I. \& Bosch, E. G. T. Phase contrast scanning transmission electron microscopy imaging of light and heavy atoms at the limit of contrast and resolution. Sci. Rep. 10, 1038 (2018).

32. Corrigan, D. The catalysis of the oxygen evolution reaction by iron impurities in thin film nickel oxide electrodes. J. Electrochem. Soc. 134, 377-384 (1987).

33. Li, X., Walsh, F. C. \& Pletcher, D. Nickel based electrocatalysts for oxygen evolution in high current density, alkaline water electrolysers. Phys. Chem. Chem. Phys. 13, 1162-1167 (2011).

34. Trotochaud, L., Ranney, J. K., William, K. N. \& Boettcher, S. W. Solution-cast metal oxide thin film electrocatalysts for oxygen evolution. J. Am. Chem. Soc. 134, 17253-17261 (2012).

35. Trotochaud, L., Young, S. L., Ranney, J. K. \& Boettcher, S. W. Nickel-iron oxyhydroxide oxygen-evolution electrocatalysts: the role of intentional and incident iron incorporation. J. Am. Chem. Soc. 136, 6744-6753 (2014).

36. Friebel, D. et al. Identification of highly active $\mathrm{Fe}$ sites in $(\mathrm{Ni}, \mathrm{Fe}) \mathrm{OOH}$ for electrocatalytic water splitting. J. Am. Chem. Soc. 137, 1305-1313 (2015).

37. Görlin, M. et al. Oxygen evolution reaction dynamics, faradic charge efficiency, and the active metal redox states of $\mathrm{Ni}-\mathrm{Fe}$ oxide water splitting electrocatalysts. J. Am. Chem. Soc. 138, 5603-5614 (2016).

38. $\mathrm{Li}$, N. et al. Influence of iron doping on tetravalent nickel content in catalytic oxygen evolving films. Proc. Natl Acad. Sci. USA 114, 1486-1491 (2017).

39. Goldsmith, Z. K. et al. Characterization of NiFe oxyhydroxide electrocatalysts by integrated electronic structure calculations and spectroelectrochemistry. Proc. Natl Acad. Sci. USA 114, 3050-3055 (2017).

40. Stevens, M. B., Trang, C. D. M., Enman, L. J., Deng, J. \& Boettcher, S. W. Reactive $\mathrm{Fe}$-sites in $\mathrm{Ni} / \mathrm{Fe}$ (oxy)hydroxide are responsible for exceptional oxygen electrocatalysis activity. J. Am. Chem. Soc. 139, 11361-11364 (2017).

41. Cai, Z. et al. Introducing $\mathrm{Fe}^{2+}$ into nickel-iron layered double hydroxide: local structure modulated water oxidation activity. Angew. Chem. Int. Ed. 57, 9392-9396 (2018).

42. Gauquelin, N. et al. Atomically resolved EELS mapping of the interfacial structure of epitaxially strained $\mathrm{LaNiO}_{3} / \mathrm{LaAlO}_{3}$ superlattices. Phys. Rev. B 90, 195140 (2014).

43. Lacorre, P. et al. Synthesis, crystal structure, and properties of metallic $\mathrm{PrNiO}_{3}$ : comparison with metallic $\mathrm{NdNiO}_{3}$ and semiconducting $\mathrm{SmNiO}_{3} . J$ Solid State Chem. 91, 225-237 (1991).

44. Stevens, M. B. et al. Measurement techniques for the study of thin film heterogeneous water oxidation electrocatalysts. Chem. Mater. 29, 120-140 (2017).

45. Ishikawa, R. et al. Direct imaging of hydrogen-atom columns in a crystal by annular bright-field electron microscopy. Nat. Mater. 10, 278-281 (2011).

46. Findlay, S. D., Azuma, S., Shibata, N., Okunishi, E. \& Ikuhara, Y. Direct oxygen imaging within a ceramic interface, with some observations upon the dark contrast at the grain boundary. Ultramicroscopy 111, 285-289 (2011).

47. Kim, J. H. et al. Atomic-scale observation of oxygen substitution and its correlation with hole-transport barriers in $\mathrm{Cu}_{2} \mathrm{ZnSnSe}_{4}$ thin-film solar cells. Adv. Energy Mater. 6, 1501902 (2016).

48. Kim, H. -S., Jang, A., Choi, S. -Y., Jung, W. \& Chung, S. -Y. Vacancy-induced electronic structure variation of acceptors and correlation with proton conduction in perovskite oxides. Angew. Chem. Int. Ed. 55, 13499-13503 (2016).

49. Byeon, P. et al. Atomic-scale observation of $\mathrm{LiFePO}_{4}$ and $\mathrm{LiCoO}_{2}$ dissolution behavior in aqueous solutions. Adv. Funct. Mater. 28, 1894564 (2018).

50. Byeon, P., Lee, H. J., Choi, J. W. \& Chung, S. -Y. Atomic-scale direct identification of surface variations in cathode oxides for aqueous and nonaqueous Li-ion batteries. ChemSusChem 12, 787-794 (2019).

51. Zhou, D. et al. Sample tilt effects on atom column position determination in ABF-STEM imaging. Ultramicroscopy 160, 110-117 (2016).

52. Gao, P. et al. Picometer-scale atom position analysis in annular bright-field STEM imaging. Ultramicroscopy 184, 177-187 (2018).
53. Jahn, H. A. \& Teller, E. Stability of polyatomic molecules in degenerate electronic states; I-Orbital degeneracy. Proc. R. Soc. Lond., Ser. A 161, 220-235 (1937)

54. $\mathrm{Li}, \mathrm{H}$. et al. Degree of geometric tilting determines the activity of $\mathrm{FeO}_{6}$ octahedra for water oxidation. Chem. Mater. 30, 4313-4320 (2018).

55. Yamada, I. et al. Synergistically enhanced oxygen evolution reaction catalysis for multielement transition-metal oxides. ACS Appl. Energy Mater. 1, 3711-3721 (2018).

56. Stoerzinger, K. A., Qiao, L., Biegalski, M. D. \& Shao-Horn, Y. Orientationdependent oxygen evolution activities of rutile $\mathrm{IrO}_{2}$ and $\mathrm{RuO}_{2}$. J. Phys. Chem. Lett. 5, 1636-1641 (2014).

57. Huang, L. -F., Hutchison, M. J., Santucci, R. J. Jr., Scully, J. R. \& Rondinelli, J. M. Improved electrochemical phase diagrams from theory and experiment: The Ni-water system and its complex compounds. J. Phys. Chem. C. 121, 9782-9789 (2017).

58. McCrory, C. C. L., Jung, S., Peters, J. C. \& Jaramillo, T. F. Benchmarking heterogeneous electrocatalysts for the oxygen evolution reaction. J. Am. Chem. Soc. 135, 16977-16987 (2013).

59. Gou, G., Grinberg, I., Rappe, A. M. \& Rondinrlli, J. M. Lattice normal modes, and electronic properties of the correlated metal $\mathrm{LaNiO}_{3}$. Phys. Rev. B 84, 144101 (2011).

60. Mosey, N. J., Liao, P. \& Carter, E. A. Rotationally invariant $a b$ initio evaluation of Coulomb and exchange parameters for DFT $+\mathrm{U}$ calculations. J. Chem. Phys. 129, 014103 (2008).

61. He, J. \& Franchini, C. Screened hybrid functional applied $3 d^{0} \rightarrow 3 d^{8}$ transitionmetal perovskites $\mathrm{LaMO}_{3}(M=\mathrm{Sc}-\mathrm{Cu})$ : influence of the exchange mixing parameter on the structural, electronic, and magnetic properties. Phys. Rev. B 86, 235117 (2012)

62. Mulliken, R. S. Electronic population analysis on LCAO-MO molecular wave functions. I. J. Chem. Phys. 28, 1833-1840 (1955).

63. Chung, S. -Y., Kim, Y. -M., Choi, S. -Y. \& Kim, J. -G. Real-time observation of crystal evaporation in a metal phosphate at high temperature. J. Am. Chem. Soc. 135, 7811-7814 (2013).

\section{Acknowledgements}

This work was supported by the National Research Foundation of Korea (NRF), grant number 2018R1A2B2006133. We gratefully acknowledge the invaluable assistance of Drs. Ioannis Alexandrou, Sorin Lazar, and Byung-Kyu Park at Thermo Fisher Scientific with the iDPC-STEM.

\section{Author contributions}

S.-Y.C. conceived and designed the project, carried out the STEM analysis and the DFT calculations, and wrote the paper. J.B. carried out all the experiments, electrochemical measurements, and specimen preparation. H.B.B. performed the STEM observations and contributed the acquisition of image data. All the authors discussed the results and commented on the manuscript.

\section{Additional information}

Supplementary Information accompanies this paper at https://doi.org/10.1038/s41467019-10838-1.

\section{Competing interests: The authors declare no competing interests.}

Reprints and permission information is available online at http://npg.nature.com/ reprintsandpermissions/

Peer review information: Nature Communications thanks Marcel Risch, Wu Zhou and the other, anonymous, reviewer(s) for their contribution to the peer review of this work.

Publisher's note: Springer Nature remains neutral with regard to jurisdictional claims in published maps and institutional affiliations.

Open Access This article is licensed under a Creative Commons Attribution 4.0 International License, which permits use, sharing, adaptation, distribution and reproduction in any medium or format, as long as you give appropriate credit to the original author(s) and the source, provide a link to the Creative Commons license, and indicate if changes were made. The images or other third party material in this article are included in the article's Creative Commons license, unless indicated otherwise in a credit line to the material. If material is not included in the article's Creative Commons license and your intended use is not permitted by statutory regulation or exceeds the permitted use, you will need to obtain permission directly from the copyright holder. To view a copy of this license, visit http://creativecommons.org/ licenses/by/4.0/.

(C) The Author(s) 2019 